\title{
Short communication
}

\section{Chirurgischer Eingriff gegen die Übervölkerung: Professor Weinholds Vorhaut-Infibulation}

Christoph Mörgeli

\section{SUMMARY}

One of the most bizarre and forgotten suggestions to have developed from the discussion of pauperism in the 19th century was the infibulation of the impoverished men. A communication in 1827 from the Halle professor of surgery, Carl August Weinhold (1782-1829), raised indignation and caused ironic as well as angry reactions. Despite such a heated response, Weinhold's proposed method of population control soon fell into oblivion, a fate considered by his contemporaries as "highly desirable".

\section{ZuSAMMENFASSUNG}

Zu den skurrilsten, heute kaum mehr bekannten Vorschlägen im Rahmen der wissenschaftlichen Pauperismus-Diskussion der ersten Hälfte des 19. Jahrhunderts gehört die Infibulation des männlichen, mittellosen Bevölkerungsteils. Eine entsprechende Schrift des Hallenser Chirurgieprofessors Carl August Weinhold (1782-1829) erregte 1827 die Gemüter und sorgte für ironische, aber auch zornige Reaktionen. Die von Weinhold entwickelte Massnahme gegen die Übervölkerung fiel indessen bald in völlige Vergessenheit, wie dies schon die meisten seiner Zeitgenossen für «höchst wünschenswert» gehalten hatten.

«Das männliche Geschlecht, von welchem als der aktiven Seite der Menschheit aller ungesetzliche Unfug zur Befriedigung einer nur tierischen Lust ausgeht, muss von nun an in weit schärfere Aufsicht als bisher genommen, ja es ihm völlig unmöglich gemacht werden, ein Wesen in die Welt zu setzen, welches zu ernähren und zu erziehen manche oft weder die Mittel noch den 
guten Willen haben ${ }^{1}$.» Solches steht in der Schrift «Von der Übervölkerung in Mittel-Europa und deren Folgen auf die Staaten und ihre Zivilisation» von 1827. Der Autor wird noch konkreter: «Ich schlage demnach als eine allgemeine und dringend notwendige Massregel eine Art von unauflöslicher Infibulation mit Verlötung und metallischer Versiegelung vor, welche nicht anders als nur gewaltsam geöffnet werden kann ${ }^{2}$.» Diese Art Infibulation habe ihm schon bei mehreren Individuen, die sich durch Onanie in eine fast unheilbare Nervenschwäche versetzt hätten, die trefflichsten Dienste geleistet. Die Operation sei leicht und nicht schmerzhafter als die Durchbohrung der Ohren zum Anbringen von Ohrringen: «Die Vorhaut wird nämlich vorgezogen und zwischen ein Paar durchlöcherte Metallplatten sanft eingeklemmt, damit das Durchstechen einer hohlen Nadel, in welcher sich ein vier bis fünf Zoll langer Bleidraht befindet, kaum gefühlt werden kann.» Sobald der Draht durchgezogen sei, müsse man ihn biegen und die beiden Endstücke mit einem Lötkolben zusammenschmelzen. Nach Erkalten der verlöteten Stelle werde ein kleiner Metallstempel aufgedrückt und dieser in Verwahrung genommen. «Es wird hierdurch ganz unmöglich, die Infibulation heimlich zu eröffnen und den Stempel wieder zu schliessen, ohne dass es nicht bei der nächsten Untersuchung entdeckt werden sollte ${ }^{3}$.» Der Eingriff muss - immer nach den Vorstellungen des Autors - bei den Männern ab dem 14. Altersjahr vorgenommen werden. Die Fibel wird ihnen erst gelöst, wenn sie imstande sind, ihre Nachkommen anständig zu ernähren; sie verbleibt all jenen zeitlebens, denen dieser Nachweis niemals gelingt. Unter die Massnahme fallen insbesondere die Bettler und Kranken, sämtliche Dienstboten, Gesellen und Lehrlinge sowie alle unverheirateten Militärpersonen «in den unteren Graden». Wegen der Gleichheit aller Staatsbürger kann die vornehme, oft sehr ausgelassene Jugend der gehobenen Stände nicht befreit werden, sofern sie die Grenzen der Sittlichkeit überschreitet, sondern muss sich «mit einigen Modifikationen» den gleichen Gesetzen unterwerfen.

Die regelmässige Kontrolle über die versiegelten Infibulationsringe gebührt einer gerichtlich-ärztlichen Behörde. Eine eigenmächtige Öffnung wird bei Jugendlichen mit der Rute, bei Erwachsenen mit der Trittmühle bestraft, «welche am besten geeignet sein dürfte, den Überschuss wollüstiger Kraft aus den Zeugungswerkzeugen in die arbeitsscheuen Arme und Beine zu ziehen». Im Wiederholungsfalle wirft man die Schuldigen bei Wasser und Brot ins Gefängnis, damit die Gesellschaft für immer vor der Gefahr verschont bleibe, «durch die gesetzwidrigen Begierden leichtsinniger und liederlicher Menschen in Verarmung zu versinken ${ }^{4} »$. 


\section{«Durch schnöde Lust der Sünde geboren»}

$\mathrm{Zu}$ solch drakonischen Massnahmen riet 1827 weder ein weltfremder Phantast noch ein grausamer Sadist. Verfasser der «dem königlich-preussischen hohen Staatsministerium ehrfurchtsvoll überreichten» Schrift war Carl August Weinhold, Doktor der Philosophie, Medizin und Chirurgie, ordentlicher Professor an der Universität zu Halle-Wittenberg, königlich-preussischer Regierungs- und Medizinalrat sowie Ritter des roten Adlerordens dritter Klasse. Weinhold wurde 1782 in Meissen geboren und trat schon im 16. Altersjahr als Kompaniechirurg ins sächsische Heer ein. Danach studierte und doktorierte er in Wittenberg. Weinhold wirkte als Arzt und Wundarzt in Dresden, wo er eine Professur für Arzneimittellehre versah, und später als beamteter Medizinalrat in Merseburg. Seit 1817 stand er der chirurgischen und augenärztlichen Klinik in Halle als Direktor vor ${ }^{5}$. Er befasste sich anlässlich einer Reise nach England mit dem Arbeiterelend in den Fabrikstädten. Durch seine berufliche Tätigkeit - so betonte Weinhold später - sah er Tag für Tag unendliches Leid in Häusern und Hütten: «Wendet euer Angesicht auf die sterbenden Säuglinge und Unmündigen, welche arme Mütter für einige Groschen in Erziehung und Kost geben müssen; sehet ihr mattes Auge, ihren sterbenden Blick, leset in ihnen die Anklage gegen die, welche sie durch schnöde Lust der Sünde geboren werden liessen ${ }^{6}$.»

In hohem Pathos und in religiös-philosophischer Beweisführung sah er in der Übervölkerung nicht göttliches Naturgesetz, sondern allein den «viehischen Missbrauch» der gottgegebenen Freiheiten durch den Menschen. Seit jeher müsse die Vernunft ausgeschaltet bleiben, wenn es um die Befriedigung des mächtigsten Triebes gehe. Pflicht aller christlichen Staaten sei es daher, praktische Massnahmen in die Tat umzusetzen, statt kaltherzige Bevölkerungsstatistiken zu führen.

Weinholds Vorschläge, so neuartig und totalitär sie klingen mögen, sind natürlich im Kontext der damals sehr lebhaft geführten PauperismusDiskussion zu betrachten. Der Chirurg in Halle stand mit seiner Angst vor der Übervölkerung keineswegs allein. Viele Intellektuelle beschäftigte die Erkenntnis, dass sich die Menschen in Europa rascher vermehrten als die zu ihrer Erhaltung erforderlichen Nahrungsquellen. So richtig ins öffentliche Bewusstsein kam die rasant ansteigende Bevölkerungskurve im beginnenden 19. Jahrhundert: Übervölkerung, Industrialisierung und der Zerfall der alten Sozialordnung standen einer stagnierenden Landwirtschaft gegenüber. Spätestens nach der Hungersnot von 1816/17 konnte niemand mehr 
die Augen vor dem Phänomen Massenarmut verschliessen. Politiker, Geistliche, Ökonomen, Pädagogen und Philanthropen bemühten sich um Lösungsvorschläge. Diese reichten von der Erziehung zum Umgang mit der Armut eines Pestalozzi bis zur gerechten Verteilung der Güter nach der Vorstellung der Frühsozialisten, von der mehr oder weniger freiwilligen Enthaltsamkeit eines Malthus bis zur Errichtung von Arbeitshäusern oder der hier geschilderten chirurgischen Zwangsmassnahme Weinholds. Viele sahen das Heilmittel in der Auswanderung nach Übersee, andere vertrauten auf den technisch-industriellen Fortschritt und auf die menschliche Erfindungsgabe ${ }^{7}$.

\section{«Die Broschüre macht Sensation»}

Die Infibulation von Männern war als mechanische Massnahme zur Verhinderung des Beischlafs nicht neu. Der medizinische Autor Celsus hat die Operation schon im 1. Jahrhundert n. Chr. genauestens beschrieben. Auch die Dichter Juvenal (6. Satire) und Martial (Epigramme VII, 81) befassten sich mit der Infibulation, die den römischen Schauspielern und Sängern zur Erhaltung der Stimme verholfen haben soll ${ }^{8}$. Der Verschluss der Vorhaut bei Athleten, wie er auf antiken Bildwerken überliefert ist, hatte wohl den ästhetisch-moralischen Zweck, die Entblössung der Eichel zu verhindern. Die Onaniehysterie des ausgehenden 18. Jahrhunderts gab dem von einigen Ärzten sehr empfohlenen Eingriff neuen Auftrieb ${ }^{9}$.

Weinholds Vorschlag einer allgemeinen Infibulation fand sofort grossen publizistischen Widerhall. Die meisten Zeitgenossen äusserten sich skeptisch bis schroff ablehnend. «Die Broschüre macht Sensation, alle Welt liest sie», schrieb in Weimar der Genfer Prinzenerzieher Frédéric Soret. Sein Hofratskollege und Freund Johann Wolfgang von Goethe liess sich die Bevölkerungslisten des Grossherzogtums vorlegen, beurteilte die Situation als wenig dramatisch und machte sich über Weinholds Idee lustig. In Halleso behauptete Soret - hätten die Studenten ihrem Professor die Fensterscheiben eingeworfen; man erzählte ihnen zur Beruhigung, Weinhold habe in seiner Jugend einem argwöhnischen Ehemann helfen wollen, die Bevölkerung Italiens zu vermehren; der betrogene Ehemann habe ihn in flagranti ertappt und zum Eunuchen gemacht ${ }^{10}$.

Am 24. Juni 1827 kam es in Halle zu einem «unruhigen Auftritt». Wie der Regierungsbevollmächtigte der Universität seinem Ministerium mitteilte, 
rief Weinholds Infibulationsvorschlag bei den Studenten keine Erbitterung, aber Spott und Lachen hervor. Ein ansehnlicher Menschenhaufen versammelte sich unter «Pfeifen und Pochen» vor dessen Haus; vereinzelte riefen «Vivat die Plombage!» Auch ging das Gerücht, der Professor sei ausserhalb der Stadt von gemeinen Leuten und Handwerksburschen grob zur Rede gestellt und wacker beschimpft worden ${ }^{11}$.

Anfangs Juli 1827 erschien eine Gegenschrift zur «Weinholdschen Übervölkerung Mittel-Europas»; hinter dem Pseudonym «Ernst Wahrhold» verbarg sich wahrscheinlich ein Hallenser Theologe. Weinholds Vorschlag schrieb Wahrhold - sei bei aller Lächerlichkeit doch zu sehr gegen jedes bürgerliche und christliche Recht, als dass er mit Stillschweigen übergangen werden dürfte. «So möchte wohl dem Sklavendienst der Wollust noch ein anderer, viel ärgerer hinzukommen, nämlich die Abhängigkeit des ganzen Menschenwesens von einem bisschen Lötblech.» Wozu bedürfe es dann noch des Mahnens und Unterrichtens durch Eltern, Lehrer oder Pfarrer, wozu guter Bücher oder geistreicher Unterhaltung? Wie könne es noch zu einer zarten, natürlichen und unschuldigen Begegnung der Geschlechter kommen? Lieber möchte man die Männer gleich kastrieren, denn der Kastrierte wisse von seiner Natur nichts mehr, der Verfibelte trage sie stets am Sklavenbande. Die die gequälten Männer umgebenden Frauen würden nicht fehlen und «das Feuer wie die Hölle unterhalten, dass Durchbrüche über Durchbrüche geschähen, alle Ruten zerschlügen und mehr Trittmühlen gemacht werden müssten, als Bretter im Lande sind ${ }^{12}$ ». Als schreiendes Unrecht empfand der unbekannte Autor, dass junge, ehrbare Dienstboten und kräftige Soldaten gefibelt würden, während reiche, alte Lüstlinge den Frauen mit noch mehr Erfolg nachstellten, als sie es bisher schon täten. Den wahrlich genug geplagten Armen und Kranken wolle man den Nachwuchs verwehren, dabei hätten gerade sie oft die gesündesten, kräftigsten Kinder. «Und zeugen sie kranke Kinder, so duldet sie Gott auch und gönnt ihnen die Luft des kurzen Lebens ${ }^{13}$.»

\section{«Wahre Skandala»}

Seit Juli 1827 lieferte Weinholds Infibulation auch Gesprächsstoff innerhalb der Medizinischen Fakultät der Universität Halle. Unter den Professoren herrschte seit Jahren unvorstellbarer Streit. Carl Heinrich Dzondi (17701835) war 1817 auf Betreiben von Friedrich Meckel (1781-1833) wegen 
angeblich frankophiler Einstellung als Chirurg abgesetzt worden, führte aber weiterhin eine vielbesuchte Klinik in Halle, was zu Reibungen mit seinem Amtsnachfolger Weinhold führte. Der Internist Peter Krukenberg (1788-1865) riet den Studenten offen, ausschliesslich seine eigenen Klinika zu besuchen ${ }^{14}$. Curt Sprengel (1766-1833), verdient um Botanik und Medizingeschichte, war seinerseits ein Einzelgänger und wechselte die Parteien nach Lust und Laune. Als «bellum omnium contra omnes» bezeichnete der Medizinhistoriker Julius Rosenbaum (1807-1874) die Hallenser Zustände ${ }^{15}$. Der grosse Chirurg Louis Stromeyer (1804-1876) hatte noch 1875 bei der Niederschrift seiner Memoiren den penetranten Braunkohlegeruch der Stadt Halle in der Nase. «Dieser infernalische Geruch haftet an allen Kleidern und scheint auch bis in die Gemüter zu dringen, deren säuerlichbrenzlige Stimmung die Streitigkeiten der Professoren verewigt ${ }^{16} . \nu$ Nach dem zynischen Stromeyer seien die chirurgischen Leistungen Weinholds in der pathologischen Universitätssammlung zu bewundern; auch mache er seinem Namen alle Ehre, da Stromeyer ihn einst vergeblich in seiner Wohnung gesucht, jedoch im Wirtshaus gefunden habe ${ }^{17}$. Ähnlich lautete das Gutachten des Berliner Chirurgen Johann Nepomuk Rust (1775-1840), der 1824 im Auftrag des preussischen Kultusministers ein Gutachten über die Hallenser Zustände verfassen musste. Rust mochte «Freund oder Feind, Jung oder Alt, Vorgesetzte oder Untergebene» fragen, er erfuhr von allen Seiten, dass «Professor Weinhold weder als Lehrer noch als Arzt irgendwelches Vertrauen besitze» und dass «seine Vorlesungen wahre Skandala» wären. Auch gehe er roh und unbeholfen mit den Kranken um und operiere «nicht wie ein Wundarzt, sondern wie ein Schlächter». Den Fakultätsprüfungen wohnte er überhaupt nicht bei, da er kein Latein konnte und fürchten musste, sich vor den Kollegen zu blamieren. Dafür zeichnete er sich aus durch seine Neigung zur Charlatanerie und zur Lügenhaftigkeit, überhaupt durch einen «unerträglichen, arroganten und zanksüchtigen Charakter». Rust musste sich dem allgemeinen Urteil anschliessen, wonach Weinhold kein "praktischer operativer Heilkünstler» sondern weiter nichts als ein «phantastischer Schriftsteller» war. Es sei einer der grössten Missgriffe, Herm Weinhold zu einem Professor der Chirurgie ernannt und ihm die Leitung eines solchen Instituts übergeben zu haben. Man solle zusehen, wie man ihn auf irgendeine Weise loswerden könne ${ }^{18}$.

Drei Jahre später schien der Zeitpunkt gekommen. Die Fakultätskollegen nahmen mündlich und schriftlich gegen Weinhold und seine Infibulation Stellung. Sie legten ihm nahe, einen halbjährigen Urlaub anzutreten. 
Ende Juli 1827 erbat er sich die Hilfe König Friedrich Wilhelms III. von Preussen. Der Monarch erliess die Anordnung, dass die Rügen nicht in Weinholds Entfernung von der Professur gipfeln durften. In Leipzig veröffentlichte Weinhold vier weitere Kampfschriften über den Missbrauch der Zeugung und über das Gleichgewicht der Bevölkerung. Seine Broschüren wurden immer umfangreicher, weitschweifiger und geschwätziger, wobei er sich zunehmend weniger mit dem Gegenstand der Übervölkerung als mit seinen zahlreichen publizistischen Feinden herumschlug ${ }^{19}$.

\section{Besuch zweier Zürcher Ärzte bei Weinhold}

Während ihren viereinhalbjährigen medizinischen Studienreisen durch Europa weilten die jungen Zürcher Ärzte Conrad Meyer-Hofmeister (18071881) und Leonhard von Muralt (1806-1891) im Mai 1829 in Halle ${ }^{20}$. Weinhold kannte Meyer-Hofmeisters Vater, Dr. med. und Spitalarzt Ludwig Meyer (1782-1851), aus der gemeinsamen Studienzeit in Würzburg und lud daher die Zürcher zum Essen ein. Meyer-Hofmeister notierte in seinem Reisebericht über Professor Weinhold: «Sein Witz und seine Schmähsucht übersteigt fast alle Begriffe, und da er sehr gut schreibt, so greift er seine Feinde gewöhnlich derb und gründlich in öffentlichen Schmähschriften an, worin man seine Satirik bewundern muss. Er spricht jetzt wenig mehr von der Infibulation, doch scheint er noch nicht ganz von dieser Verirrung seines Geistes geheilt zu sein; vielleicht, dass der Grund dieser fast verrückten Idee in seiner Konstitution gefunden werden könnte. Er sei nämlich unverheiratet, hat zwar ein langes Corpus, besonders seine Arme und Füsse sind sehr lang, wogegen sein Hals und Kopf klein sind. Daneben hat er keine Spur von einem Bart, eine mehr weibliche als männliche Stimme, und Dr. Moser ${ }^{21}$ behauptete wirklich, dass seine Geschlechtsteile übel gebildet seien. An dem Abend, da wir bei ihm waren, schimpfte er fast beständig über eine neue Schikane, welche ihm von Sprengel und Krukenberg angezettelt worden sei, indem diese nämlich beim Ministerium bewirkt hatten, dass ein Dr. Blasius ${ }^{22}$ nach Halle kommen sollte, um Chirurgie zu lesen und einen Operationskurs zu halten. Allein, Weinhold will ihm weder Instrumente noch Kadaver abtreten, und Rust befiehlt von Berlin aus, dies müsse geschehen. So entsteht wieder ein Streit, da Weinhold sich auf seine ihm beim Antritte dieser Stelle bewilligten Bedingungen stützte. 〈Obschon ich〉, sagte er, 〈gerne von Halle wegginge, so will ich nun gerade diesen Herren Trotz bieten. Ich 
wende mich nicht mehr ans Ministerium, sondern direkt an den König, und hilft mir dieser nicht, so gebe ich die ganze Schikane öfentlich im Druck heraus ${ }^{23}$ ')!.

Weinhold war zweifellos psychisch angeschlagen und wusste um seinen Zustand. «Nun bleibt also nichts weiter übrig», schrieb er zuletzt, «als etwas konzentriertes Mandelwasser in einer Tasse Kaffee, um auch die physische Existenz zu vernichten ${ }^{24}$.» Weinhold starb 1829 im Alter von erst 47 Jahren, möglicherweise durch Selbstmord. Sein Infibulationsvorschlag verfiel der «wohlverdienten Vergessenheit», wie dies das preussische Ministerium 1827 für «höchst wünschenswert» gehalten hatte ${ }^{25}$. Oder wenigstens beinahe: 1930 räumte ein Mitarbeiter der dem Nationalsozialismus nahestehenden «Münchner Medizinischen Wochenschrift» ein, dass man bei Weinhold abgesehen vom Infibulationsvorschlag - manche Ideen finde, denen auch moderne Sozialpolitiker und Eugeniker zustimmen könnten. «Ohne Parteisozialist zu sein», zeige dieser ein warmes soziales Empfinden. «Was Weinhold über die Grundlage der Ehe, über die Bezähmung der Triebe mit Rücksicht auf die Gesellschaft schreibt, vertritt die heutige Rassenhygiene ${ }^{26} . »$ 


\section{Anmerkungen}

1 C. A. Weinhold: Von der Überbevölkerung in Mittel-Europa und deren Folgen auf die Staaten und ihre Zivilisation, Halle 1827, S.31.

2 Ibid., S. 32.

3 Ibid., S. $32 \mathrm{f}$.

4 Ibid., S. $33 \mathrm{f} ., 46 \mathrm{f}$.

5 Intelligenzblatt der Allgemeinen Literatur-Zeitung 104, 1829, S. 842 f. Neuer Nekrolog der Deutschen, Bd. 7.2, 1829, S.677-681. Salzburger medizinisch-chirurgische Zeitung 3, 1830, S.236. A.C.P. Callisen: Medizinisches Schriftsteller-Lexikon, Bd.33, 1845, S.252. Allgemeine Deutsche Biographie, Bd.41, 1896, S. 504 f. (J.Pagel). J. Hirschberg: Geschichte der Augenheilkunde, Graefe-Saemisch 14.2, 2. Aufl., Leipzig 1911, S.178. Biographisches Lexikon der hervorragenden Ärzte aller Zeiten und Völker, Bd.5, Berlin/Wien 1934, S.882 (J.Pagel).

6 Vgl. Anm. 1, S. 30.

7 Neuere Werke zum Pauperismus: Die Eigentumslosen, der deutsche Pauperismus und die Emanzipationskrise in Darstellung und Deutungen der zeitgenössischen Literatur, bearb. und hrsg. von C. Jantke und D. Hilger, Freiburg 1965. Etudes sur l'histoire de la pauvreté, 2 vols, sous la direction de M. Mollat, Paris 1974. C. Sachsse, F. Tennstedt: Geschichte der Armenfürsorge in Deutschland, vom Spätmittelalter bis zum Ersten Weltkrieg, Stuttgart 1980. W. Fischer: Armut in der Geschichte, Erscheinungsformen und Lösungsversuche der «Sozialen Frage» in Europa seit dem Mittelalter, Göttingen 1982. S. Woolf: The poor in Western Europe in the eighteenth and nineteenth centuries, London/New York 1986. B. Geremek: Geschichte der Armut, Elend und Barmherzigkeit, München/Zürich 1988.

8 J. N. Rust: Theoretisch-praktisches Handbuch der Chirurgie, Bd.9, Berlin/Wien 1833, S.584-587. L. Stieda: Die Infibulation bei Griechen und Römern, in: Anatomische Hefte, 1. Abt., 19, Wiesbaden 1902, H.62, S.233-308 (mit ausführlicher Literatur!). W. Schönfeld: Zweck der Infibulation und Vorhautplastik der Alten, in: Hautarzt 5, 1954, S. 273 276. A.Pillokat: Infibulation in der Gegenwart, in: Hautarzt 5, 1954, S. 515.

9 S. G. Vogel: Unterricht für Eltern und Erzieher, wie das unglaublich gemeine Laster der zerstörenden Selbstbefleckung am sichersten zu entdecken, zu verhüten und zu heilen sei, Stendal 1786, 2. Aufl. 1789. Neben Weinhold (Anm. 1, S. 32) hat auch Carl Ferdinand von Graefe (1787-1840) die Operation durchgeführt, s. J. N. Rust: Theoretisch-praktisches Handbuch der Chirurgie, Bd.9, Berlin/Wien 1833, S. 587. Ablehnend äussert sich neben Rust auch G. Dupuytren wegen eines Falls «einer durch Infibulation entstandenen szirrhösen Entartung der Vorhaut», s. Répertoire général d'anatomie et de physiologie pathologiques et de clinique chirurgicale (Paris), tome 4, 1828, p. 204. Vgl. auch Hamburgisches Magazin der ausländischen Literatur der gesamten Heilkunde und Arbeiten des ärztlichen Vereins zu Hamburg, Bd.16, Nov./Dez.1828, S.292-294.

10 F. Soret: Zehn Jahre bei Goethe, Erinnerungen an Weimars klassische Zeit 1822-1832, hrsg. von H. H. Houben, Leizpig 1929, S.214f.

11 Bericht des ausserordentlichen Regierungsbevollmächtigten Georg Hartmann von Witzleben (1766-1841) ans preussische Ministerium der geistlichen, Unterrichts- und Medizinal- 
angelegenheiten vom 26.6.1827. Zitiert nach H.-T. Koch: Karl August Weinhold (17821829) und sein Infibulationsvorschlag, in: Acta Historica Leopoldina 2, Leipzig 1965, S. 185 .

12 E. Wahrhold: Die Weinholdsche Übervölkerung Mittel-Europas, Halle 1827, S.4, 7 f., 9.

13 Ibid., S. 21.

14 C. Meyer-Hofmeister: Beschreibung der medizinisch-chirurgischen Anstalten in Hamburg, Halle, Jena, Weimar, Bamberg und München, o. J. (1828, 1829), Mskr., Familienbesitz, S. 56 .

15 J. Rosenbaum: Neun Jahre aus dem Leben eines Privatdozenten, Leipzig 1847, S. 37.

17 Ibid., S. 208, 210.

18 H.-T. Koch: Ein Gutachten über die Medizinische Fakultät Halle von Johann Nepomuk Rust aus dem Jahre 1824, in: Acta Historica Leopoldina 2, 1965, S. 162-171.

19 C. A. Weinhold: Von der überwiegenden Reproduktion des Menschenkapitals gegen das Betriebskapital und die Arbeit in den zivilisiertesten europäischen Ländern, Leipzig 1828. Ders.: Über die Population und die Industrie, oder kritischer Beweis, dass die Bevölkerung in hochkultivierten Ländern den Gewerbefleiss stets übereile, Leipzig 1828. Ders.: Über das menschliche Elend, welches durch den Missbrauch der Zeugung herbeigeführt wird, Leipzig 1828. Ders.: Das Gleichgewicht der Bevölkerung als Grundlage der Wohlfahrt der Gesellschaft und der Familien, Leipzig 1829.

20 C. Meyer-Hofmeister: Reisetagebuch, 20.-21.5.1829, Mskr., Familienbesitz.

21 Prosektor bei J.F. Meckel. A.C.P.Callisen: Medizinisches Schriftsteller-Lexikon der jetzt lebenden Ärzte, Bd.13, 1833, S.260. Ansonsten keine weiteren Angaben bekannt.

22 Ernst Blasius (1802-1875), 1828 PD, 1830 a. o. Prof., 1834-1867 o. Prof. der Chirurgie in Halle.

23 S. Anm. 14, S. 68-70.

24 C.A. Weinhold: Das Gleichgewicht der Bevölkerung als Grundlage der Wohlfahrt der Gesellschaft und der Familien, Leipzig 1829, S. 7.

25 S. Anm. 12, S. 186.

26 (W.) Rimpau: Carl August Weinhold über den Missbrauch der Zeugung, in: Münchener medizinische Wochenschrift 77, 1930, S. 1460.

Dr. phil. Christoph Mörgeli

Konservator des Medizinhistorischen

Museums der Universität Zürich

Rämistrasse 71

CH-8006 Zürich 